\title{
Introduction from the incoming editor
}

\author{
J. Christopher Westland
}

Published online: 6 November 2014

(C) Springer Science+Business Media New York 2014

This issue of the Electronic Commerce Research journal marks a transition as I begin my tenure as Editor-in-Chief. I have participated on the Editorial Board of the journal since its inception in 2001, and have witnessed a phenomenal growth in academic interest in e-commerce. During this period I have watched electronic commerce grow from a niche industry to one central to modern business, economics and personal endeavors.

ECR was the pioneering scholarly, peer-reviewed journal specializing in e-commerce, and is today widely read and cited (2013 impact factor 1.632). But challenges loom-a proliferation of on-line options for research and publication are dramatically altering the nature of research dissemination, and placing new demands on editors and publishers. Article search and citation are now more affected by Google search placement than by traditional bibliometrics, and social feeds, including Facebook and Twitter, assert an increased influence on readers. Furthermore, international readership, particularly in emerging economies, is noticeably changing the look and content of academic work in all areas. This is only natural - it reflects the pervasiveness of Internet technologies today in all fields, including academic publishing.

ECR intends to remain at the forefront in managing these changes, with innovative stakeholder incentive systems to manage paper quality, reviewer and author buy-in, citations, bibliometrics and their alternatives such as views, downloads, social and news media. Expect the focus of ECR's research articles to evolve over the next year, as legacy papers work their way through the review system and the journal's new taxonomy and mission statement influence future submissions. We anticipate also that our Editorial and Advisory Board membership will change over the coming issues, reflecting the ongoing shift in relevant e-commerce topics. Most of this will be invisible

\footnotetext{
J. Christopher Westland ( $\square)$

University of Illinois, Chicago, USA

e-mail:westland@uic.edu
} 
to authors, but will show up in the longer run improvements in our authors' personal productivity metrics such as the h-index, as well as journal impact factors and alternative metrics. We are continually developing systems for widespread dissemination of ECR's research across multiple venues, both academic and mass-market, to increase the impact of each article we publish.

Looking towards the future, we plan to expand the breadth of our offerings to reflect emerging areas of both interest and controversy in electronic commerce. Our regular research articles will continue to comprise the majority of published work in the journal. But we will expand this by inviting shorter submissions-Research Notes - that describe interesting preliminary findings, or findings that may be seen as corollaries to published findings.

In addition to regular articles, we are soliciting three new categories of Research Note submissions-methodology, disconfirming (negative) empirical results, and under-researched, speculative and contrarian topics. Our intention is to publish short, provocative synopses of research that will interest our readers in the electronic commerce community, but which are currently underserved in the journals.

Methodology papers will synopsize innovative, unique models or methodologies of broad interest in e-commerce research. We especially encourage authors who have already published research using a new method to synopsize the method and its applications to e-commerce.

Disconfirming or negative results are underrepresented in traditional journals. As a result, many fields find their research biased towards positive findings even when these inaccurately depict reality and leave future research at risk of spending time and money replicating failure. Significant progress has been made over the last decade, particularly in medicine, towards a more balanced publication of results. ECR intends to provide a forum to bring similar balance to e-commerce research.

Our third new type of paper recognizes that established research paradigms tend to be comprised of many interrelated components. Too often, good ideas will lack one or more components required for research closure, and consequently may not be disseminated to the larger research community, slowing progress. ECR intends to provide a forum for brief, high-quality expositions of under-researched, speculative or contrarian ideas that would be of interest in industry and academe. The benefits of such accounts have long been recognized, most influentially in British mathematician I.J. Good's book The Scientist Speculates. Such papers can range from novel questions; speculations; suggestions for a novel experiment; stimulating analogies; or (rarely) frameworks and classifications.

From time to time, I will ask Advisory Board members, as well as other noted academic and industry thought leaders, to contribute essays on topics that are trending, and solicit their opinions on controversial topics within the scope of ECR. It is my hope that these short opinion pieces will inspire new and innovative research that we will eventually see on the pages of the journal.

Lastly, I welcome and encourage your suggestions, insights and ideas for the journal. Contact information along with submission guidelines and the journal's mission statement are available online at Electronic Commerce Research journal's Web site. I look forward to working with you in the days ahead. 